\title{
VIJAY GUPTA
}

\section{Certain family of Durrmeyer type operators}

\begin{abstract}
The present paper is a continuation of the earlier work of the author. Here we study the rate of convergence of certain Durrmeyer type operators for function having derivatives of bounded variation.
\end{abstract}

1. Introduction. The integral modification of the well-known Bernstein polynomials was introduced by Durrmeyer [2]. Derriennic [1] established some direct results in ordinary and simultaneous approximation for the Durrmeyer operators. The rate of convergence of the Durrmeyer operators was studied by Zeng and Chen [6]. Very recently Gupta et al. [3] considered a family of Durrmeyer type operators $P_{n, r}(f, x)$ defined in the following way. Let $p_{n, k}(x)=\left(\begin{array}{l}n \\ k\end{array}\right) x^{k}(1-x)^{n-k}$. Then

$P_{n, r}(f, x)= \begin{cases}n \sum_{k=1}^{n} p_{n, k}(x) \int_{0}^{1} p_{n-1, k-1}(t) f(t) d t+p_{n, 0}(x) f(0), & r=0, \\ \frac{n^{\underline{r}}}{(n+r-1) \frac{r-1}{n}} \sum_{k=0}^{n-r} p_{n-r, k}(x) \int_{0}^{1} p_{n+r-1, k+r-1}(t) f(t) d t, & r>0,\end{cases}$

where $r, n \in \mathbb{N}_{0}$ with $r \leq n$ and for any $a, b \in \mathbb{N}_{0}, a^{\underline{b}}=a(a-1) \cdots(a-b+1)$, $a^{\underline{0}}=1$ is the falling factorial. One can notice that the family $P_{n, r}(f, x)$, as a particular case $(r=0)$, contains the sequence $P_{n, 0}(f, x)$ introduced by Srivastava and Gupta in [5]. The rate of convergence of functions of

2000 Mathematics Subject Classification. 41A25, 41A35.

Key words and phrases. Bernstein polynomials, Durrmeyer operators, bounded variation, total variation. 
bounded variation of the Bézier variant of the special case of these operators was obtained by Gupta and Maheshwari in [4].

The operators $P_{n, r}(f, x)$ also admit the integral representation

$$
P_{n, r}(f, x)=\int_{0}^{1} K_{r, n}(x, t) f(t) d t,
$$

where the kernel $K_{n, r}(x, t)$ is given by

$$
K_{n, r}(x, t)= \begin{cases}n \sum_{k=1}^{n} p_{n, k}(x) p_{n-1, k-1}(t)+p_{n, 0}(x) \delta(t), & r=0, \\ \frac{n^{\underline{r}}}{(n+r-1) \frac{r-1}{n}} \sum_{k=0}^{n-r} p_{n-r, k}(x) p_{n+r-1, k+r-1}(t), & r>0 .\end{cases}
$$

Here, $\delta(t)$ denotes the Dirac delta function.

The aim of the present paper is to extend the study on the operators $P_{n, r}(f, x)$. Here we study the rate of convergence for these operators for functions having derivatives of bounded variation. We also present a corollary in the end, which gives the result in simultaneous approximation.

2. Auxiliary results. In the sequel we shall need several lemmas. First we will study the moments of the operators $P_{n, r}(f, x)$. For this purpose we define for any $m \in \mathbb{N}_{0}$

$$
\begin{aligned}
T_{n, r, m}(x) & =\frac{(n+r)^{\underline{r}}}{n^{\underline{\underline{r}}}} P_{n, r}\left((t-x)^{m}, x\right) \\
& = \begin{cases}n \sum_{k=1}^{n} p_{n, k}(x) \int_{0}^{1} p_{n-1, k-1}(t)(t-x)^{m} d t+(-x)^{m} p_{n, 0}(x), & r=0, \\
(n+r) \sum_{k=0}^{n-r} p_{n-r, k}(x) \int_{0}^{1} p_{n+r-1, k+r-1}(t)(t-x)^{m} d t, & r>0 .\end{cases}
\end{aligned}
$$

Lemma 1 ([3]). The following claims hold:

(i) For any $r, m \in \mathbb{N}_{0}$ and $x \in[0,1]$, the recurrence relation is satisfied

$$
\begin{aligned}
(n+m+r+1) T_{n, r, m+1}(x)= & x(1-x)\left[D T_{n, r, m}(x)+2 m T_{r, n, m-1}(x)\right] \\
& +[(m+r)-x(1+2 m+2 r)] T_{n, r, m}(x),
\end{aligned}
$$

where, for $m=0$, we denote $T_{n, r,-1}(x)=0$.

(ii) For all $r \in \mathbb{N}_{0}$ and $x \in[0,1]$,

$$
\begin{gathered}
T_{n, r, 0}(x)=1, \quad T_{n, r, 1}(x)=\frac{r-x(1+2 r)}{n+r+1}, \\
T_{n, r, 2}(x)=\frac{2 n x(1-x)+r(1+r)-2 r x(2 r+3)+2 x^{2}\left(2 r^{2}+4 r+1\right)}{(n+r+1)(n+r+2)} .
\end{gathered}
$$

(iii) For all $r, m \in \mathbb{N}_{0}$ and $x \in[0,1]$,

$$
T_{n, r, m}(x)=O\left(n^{-[(m+1) / 2]}\right) .
$$


Lemma 2 ([3]). For any $r, s \in \mathbb{N}_{0}$ and $x \in[0,1]$,

$$
D^{s} P_{n, r}(f, x)=P_{n, r+s}\left(D^{s} f, x\right) .
$$

Remark 1. For $n$ sufficiently large and $x \in(0,1)$, it can be seen from Lemma 1, that

$$
\frac{x(1-x)}{n} \leq T_{n, r, 2}(x) \leq \frac{C x(1-x)}{n},
$$

for any $C>2$.

We denote

$$
\hat{K}_{n, r}(x, t)= \begin{cases}n \sum_{k=1}^{n} p_{n, k}(x) p_{n-1, k-1}(t)+p_{n, 0}(x) \delta(t), & r=0, \\ (n+r) \sum_{k=0}^{n-r} p_{n-r, k}(x) p_{n+r-1, k+r-1}(t), & r>0 .\end{cases}
$$

$\delta(t)$ denotes the Dirac delta function.

Remark 2. For $n$ sufficiently large and $x \in(0,1)$, it can be seen from Schwarz inequality and Remark 1, that

$$
\int_{0}^{1} \hat{K}_{n, r}(x, t)|t-x| d t \leq\left[T_{n, r, 2}(x)\right]^{1 / 2} \leq \sqrt{\frac{C x(1-x)}{n}},
$$

for any $C>2$.

Lemma 3 ([3]). Let $x \in(0,1)$ and $C>2$, then for $n$ sufficiently large, we have

$$
\begin{gathered}
\lambda_{n, r}(x, y)=\int_{0}^{y} \hat{K}_{n, r}(x, t) d t \leq \frac{C x(1-x)}{n(x-y)^{2}}, \quad 0 \leq y<x, \\
1-\lambda_{n, r}(x, z)=\int_{z}^{1} \hat{K}_{n, r}(x, t) d t \leq \frac{C x(1-x)}{n(z-x)^{2}}, \quad x<z<1 .
\end{gathered}
$$

3. Rate of convergence. By $D B(0,1)$, we denote the class of absolutely continuous functions $f$ defined on $(0,1)$ which have a derivative $f^{\prime}$ on the interval $(0,1)$, coinciding a.e. with a function which is of bounded variation on every finite subinterval of $(0,1)$.

It can be observed that all functions $f \in D B(0,1)$ possess for each $c>0$ the representation

$$
f(x)=f(c)+\int_{c}^{x} \psi(t) d t, \quad x \geq c .
$$

In this section, we prove the following main theorem. 
Theorem 1. Let $f \in D B(0,1)$, and $x \in(0,1)$. Then for $C>2$ and $n$ sufficiently large, we have

$$
\begin{aligned}
& \mid \frac{(n+r)^{\underline{r}}}{n^{\underline{r}}} P_{n, r}(f, x)-f(x)-\frac{1}{2}\left(f^{\prime}\left(x^{+}\right)-f^{\prime}\left(x^{-}\right)\right) \sqrt{\frac{C x(1-x)}{n}} \\
& -\frac{1}{2}\left(f^{\prime}\left(x^{+}\right)+f^{\prime}\left(x^{-}\right)\right) \frac{r-x(1+2 r)}{n+r+1} \mid \\
& \leq \frac{C}{n x(1-x)}\left(\sum_{k=1}^{[\sqrt{n}]} \bigvee_{x-x / k}^{x+(1-x) / k}\left(\left(f^{\prime}\right)_{x}\right)+\frac{1}{\sqrt{n}} \bigvee_{x-x / \sqrt{n}}^{x+(1-x) / \sqrt{n}}\left(\left(f^{\prime}\right)_{x}\right)\right)
\end{aligned}
$$

where $\bigvee_{a}^{b} f(x)$ denotes the total variation of $f_{x}$ on $[a, b]$, and $f_{x}$ is defined by

$$
f_{x}(t)= \begin{cases}f(t)-f\left(x^{-}\right), & 0 \leq t<x \\ 0, & t=x \\ f(t)-f\left(x^{+}\right), & x<t<1\end{cases}
$$

Proof. Using the mean value theorem, we can write

$$
\begin{aligned}
\left|\frac{(n+r)^{\underline{r}}}{n^{\underline{r}}} P_{n, r}(f, x)-f(x)\right| & \leq \int_{0}^{1} \hat{K}_{n, r}(x, t)|f(t)-f(x)| d t \\
& =\int_{0}^{1}\left|\int_{x}^{t} \hat{K}_{n, r}(x, t) f^{\prime}(u) d u\right| d t .
\end{aligned}
$$

Also, we use the identity

$$
\begin{aligned}
f^{\prime}(u)= & \frac{f^{\prime}\left(x^{+}\right)+f^{\prime}\left(x^{-}\right)}{2}+\left(f^{\prime}\right)_{x}(u)+\frac{f^{\prime}\left(x^{+}\right)-f^{\prime}\left(x^{-}\right)}{2} \operatorname{sgn}(u-x) \\
& +\left[f^{\prime}(x)-\frac{f^{\prime}\left(x^{+}\right)+f^{\prime}\left(x^{-}\right)}{2}\right] \chi_{x}(u),
\end{aligned}
$$

where

$$
\chi_{x}(u)= \begin{cases}1, & u=x \\ 0, & u \neq x\end{cases}
$$

Obviously, we have

$$
\int_{0}^{1}\left(\int_{x}^{t}\left(f^{\prime}(x)-\frac{f^{\prime}\left(x^{+}\right)+f^{\prime}\left(x^{-}\right)}{2}\right) \chi_{x}(u) d u\right) \hat{K}_{n, r}(x, t) d t=0 .
$$


Thus, using above identities, we can write

$$
\begin{aligned}
& \left|\frac{(n+r)^{\underline{r}}}{n^{\underline{r}}} P_{n, r}(f, x)-f(x)\right| \\
& \quad \leq \int_{0}^{1}\left(\int_{x}^{t} \hat{K}_{n, r}(x, t)\left(\frac{\left[f^{\prime}\left(x^{+}\right)+f^{\prime}\left(x^{-}\right)\right]}{2}+\left(f^{\prime}\right)_{x}(u)\right) d u\right) d t \\
& \quad+\int_{0}^{1}\left(\int_{x}^{t} \hat{K}_{n, r}(x, t) \frac{\left[f^{\prime}\left(x^{+}\right)-f^{\prime}\left(x^{-}\right)\right]}{2} \operatorname{sgn}(u-x) d u\right) d t .
\end{aligned}
$$

Also, it can be verified that

$$
\begin{gathered}
\int_{0}^{1}\left(\int_{x}^{t} \frac{\left[f^{\prime}\left(x^{+}\right)-f^{\prime}\left(x^{-}\right)\right]}{2} \operatorname{sgn}(u-x) d u\right) \hat{K}_{n, r}(x, t) d t \\
\leq \frac{\left|f^{\prime}\left(x^{+}\right)-f^{\prime}\left(x^{-}\right)\right|}{2}\left[T_{n, r, 2}(x)\right]^{1 / 2}
\end{gathered}
$$

and

$$
\begin{gathered}
\int_{0}^{1}\left(\int_{x}^{t} \frac{1}{2}\left[f^{\prime}\left(x^{+}\right)+f^{\prime}\left(x^{-}\right)\right] d u\right) \hat{K}_{n, r}(x, t) d t \\
=\frac{1}{2}\left[f^{\prime}\left(x^{+}\right)+f^{\prime}\left(x^{-}\right)\right] T_{n, r, 1}(x) .
\end{gathered}
$$

Combining (1)-(3), we have

(4)

$$
\begin{aligned}
& \mid \frac{(n+r)^{\underline{r}}}{n^{\underline{r}}} P_{n, r}(f, x)-f(x)-\frac{1}{2}\left(f^{\prime}\left(x^{+}\right)-f^{\prime}\left(x^{-}\right)\right)\left[T_{n, r, 2}(x)\right]^{1 / 2} \\
& -\frac{1}{2}\left(f^{\prime}\left(x^{+}\right)+f^{\prime}\left(x^{-}\right)\right) T_{n, r, 1}(x) \\
& \leq\left|\int_{x}^{1}\left(\int_{x}^{t}\left(f^{\prime}\right)_{x}(u) d u\right) \hat{K}_{n, r}(x, t) d t+\int_{0}^{x}\left(\int_{x}^{t}\left(f^{\prime}\right)_{x}(u) d u\right) \hat{K}_{n, r}(x, t) d t\right| \\
& =:\left|A_{n, r}(f, x)+B_{n, r}(f, x)\right| \text {. }
\end{aligned}
$$

Making use of Remark 1 and Remark 2 in (4), we have

$$
\begin{aligned}
& \mid \frac{(n+r)^{\underline{r}}}{n^{\underline{r}}} P_{n, r}(f, x)-f(x)-\frac{1}{2}\left(f^{\prime}\left(x^{+}\right)-f^{\prime}\left(x^{-}\right)\right) \sqrt{\frac{C x(1-x)}{n}} \\
&-\frac{1}{2}\left(f^{\prime}\left(x^{+}\right)+f^{\prime}\left(x^{-}\right)\right) \frac{r-x(1+2 r)}{n+r+1} \mid \\
& \leq\left|A_{n, r}(f, x)\right|+\left|B_{n, r}(f, x)\right| .
\end{aligned}
$$


Since $\int_{a}^{b} d_{t}\left(\lambda_{n, r}(x, t)\right) \leq 1$ for all $[a, b] \subseteq[0,1]$, applying integration by parts and Lemma 3 with $y=x-x / \sqrt{n}$, we have

$$
\begin{aligned}
& \left|B_{n, r}(f, x)\right|=\left|\int_{0}^{x}\left(\int_{x}^{t}\left(f^{\prime}\right)_{x}(u) d u\right) d_{t}\left(\lambda_{n, r}(x, t)\right)\right| \\
& =\left|\int_{0}^{x} \lambda_{n, r}(x, t)\left(f^{\prime}\right)_{x}(t) d t\right| \leq\left(\int_{0}^{y}+\int_{y}^{x}\right)\left|\left(f^{\prime}\right)_{x}(t)\right|\left|\lambda_{n, r}(x, t)\right| d t \\
& \leq \frac{C x(1-x)}{n} \int_{0}^{y} \bigvee_{t}^{x}\left(\left(f^{\prime}\right)_{x}\right) \frac{1}{(x-t)^{2}} d t+\int_{y}^{x} \bigvee_{t}^{x}\left(\left(f^{\prime}\right)_{x}\right) d t \\
& \leq \frac{C x(1-x)}{n} \int_{0}^{y} \bigvee_{t}^{x}\left(\left(f^{\prime}\right)_{x}\right) \frac{1}{(x-t)^{2}} d t+\frac{x}{\sqrt{n}} \bigvee_{x-\frac{x}{\sqrt{n}}}^{x}\left(\left(f^{\prime}\right)_{x}\right)
\end{aligned}
$$

Let $u=\frac{x}{x-t}$. Then we have

$$
\begin{aligned}
\frac{C x(1-x)}{n} \int_{0}^{y} \bigvee_{t}^{x}\left(\left(f^{\prime}\right)_{x}\right) \frac{1}{(x-t)^{2}} d t & =\frac{C x(1-x)}{n x^{2}} \int_{1}^{\sqrt{n}} \bigvee_{x-\frac{x}{u}}^{x}\left(\left(f^{\prime}\right)_{x}\right) d u \\
& \leq \frac{C(1-x)}{n x} \sum_{k=1}^{[\sqrt{n}]} \bigvee_{x-\frac{x}{k}}^{x}\left(\left(f^{\prime}\right)_{x}\right)
\end{aligned}
$$

Thus

(6)

$$
\left|B_{n, r}(f, x)\right| \leq \frac{C(1-x)}{n x} \sum_{k=1}^{[\sqrt{n}]} \bigvee_{x-\frac{x}{k}}^{x}\left(\left(f^{\prime}\right)_{x}\right)+\frac{x}{\sqrt{n}} \bigvee_{x-\frac{x}{\sqrt{n}}}^{x}\left(\left(f^{\prime}\right)_{x}\right)
$$

On the other hand, we have

$(7)$

$$
\begin{aligned}
& \left|A_{n, r}(f, x)\right|=\left|\int_{x}^{1}\left(\int_{x}^{t}\left(f^{\prime}\right)_{x}(u) d u\right) \hat{K}_{n, r}(x, t) d t\right| \\
& =\mid \int_{z}^{1}\left(\int_{x}^{t}\left(f^{\prime}\right)_{x}(u) d u\right) \hat{K}_{n, r}(x, t) d t \\
& \quad+\int_{x}^{z}\left(\int_{x}^{t}\left(f^{\prime}\right)_{x}(u) d u\right) d_{t}\left(1-\lambda_{n, r}(x, t)\right) d t \mid \\
& \leq \frac{C}{n(1-x)} \int_{1}^{\sqrt{n}} \bigvee_{x}^{x+(1-x) / \sqrt{n}}\left(\left(f^{\prime}\right)_{x}\right) d u+\frac{(1-x)}{\sqrt{n}} \bigvee_{x}^{x+(1-x) / \sqrt{n}}\left(\left(f^{\prime}\right)_{x}\right) \\
& \leq \frac{C}{n(1-x)} \sum_{k=1}^{[\sqrt{n}]} \bigvee_{x}^{x+(1-x) / k}\left(\left(f^{\prime}\right)_{x}\right)+\frac{(1-x)}{\sqrt{n}} \bigvee_{x}^{x+(1-x) / \sqrt{n}}\left(\left(f^{\prime}\right)_{x}\right) .
\end{aligned}
$$

Collecting the estimates (5)-(7), and using the fact that $(1-x)^{2}+x^{2} \leq 1$, we get the required result. This completes the proof of Theorem 1. 
Furthermore, as a corollary of Theorem 1, if we take into account the differential formula given by Lemma 2 we can easily prove the following result for the derivatives of the operators $P_{n, r}$.

Corollary 1. Let $f^{(s)} \in D B(0,1)$, and $x \in(0,1)$. Then for $C>2$ and $n$ sufficiently large, we have

$$
\begin{aligned}
& \mid \frac{(n+r+s) \frac{r+s}{n}}{n \frac{r+s}{s}} D^{s} P_{n, r}(f, x)-f^{(s)}(x) \\
& \quad-\frac{1}{2}\left(D^{s+1} f\left(x^{+}\right)-D^{s+1} f\left(x^{-}\right)\right) \sqrt{\frac{C x(1-x)}{n}} \\
& \quad-\frac{1}{2}\left(D^{s+1} f\left(x^{+}\right)+D^{s+1} f\left(x^{-}\right)\right) \frac{r+s-x(1+2(r+s))}{n+r+s+1} \mid \\
& \leq \frac{C}{n x(1-x)}\left(\sum_{k=1}^{[\sqrt{n}]} \bigvee_{x-x / k}^{x+(1-x) / k}\left(\left(f^{\prime}\right)_{x}\right)+\frac{1}{\sqrt{n}} \bigvee_{x-x / \sqrt{n}}^{x+(1-x) / \sqrt{n}}\left(\left(f^{\prime}\right)_{x}\right)\right)
\end{aligned}
$$

where $\bigvee_{a}^{b} f(x)$ denotes the total variation of $f_{x}$ on $[a, b]$, and $f_{x}$ is defined by

$$
D^{s+1} f_{x}(t)= \begin{cases}D^{s+1} f(t)-D^{s+1} f\left(x^{-}\right), & 0 \leq t<x \\ 0, & t=x \\ D^{s+1} f(t)-D^{s+1} f\left(x^{+}\right), & x<t<1\end{cases}
$$

\section{REFERENCES}

[1] Derriennic, M.-M., Sur l'approximation de functions integrable sur [0,1] par des polynomes de Bernstein modifies, J. Approx. Theory 31 (1981), 323-343.

[2] Durrmeyer, J. L., Une formule d'inversion de la Transformee de Laplace, Applications a la Theorie des Moments, These de 3e Cycle, Faculte des Sciences de l' Universite de Paris, 1967.

[3] Gupta, V., López-Moreno, A. J. and Latorre-Palacios, J.-M., On simultaneous approximation of the Bernstein Durrmeyer operators, Appl. Math. Comput. 213 (1) (2009), $112-120$.

[4] Gupta, V., Maheshwari, P., Bézier variant of a new Durrmeyer type operators, Riv. Mat. Univ. Parma (6) 7 (2) (2003), 9-21.

[5] Srivastava, H. M., Gupta, V., A certain family of summation-integral type operators, Math. Comput. Modelling 37 (2003), 1307-1315.

[6] Zeng, X. M., Chen, W., On the rate of convergence of the generalized Durrmeyer type operators for functions of bounded variation, J. Approx. Theory 102 (2000), 1-12.

Vijay Gupta

School of Applied Sciences

Netaji Subhas Institute of Technology

Sector 3 Dwarka New Delhi-110078

India

e-mail: vijaygupta2001@hotmail.com

Received April 9, 2009 